\title{
Government Intervention Measures Effectively Control COVID-19 Epidemic in Wuhan, China
}

\author{
Xing XIN ${ }^{1 \dagger}$, Shu-fang $\mathrm{LI}^{1 \dagger}$, Ling $\mathrm{CHENG}^{2}$, Chang-yu LIU ${ }^{3}$, Yin-juan XIN ${ }^{4}$, Hai-long HUANG ${ }^{5}$, Rajluxmee Beejadhursing ${ }^{1}$, \\ Shao-shuai WANG ${ }^{1 \#}$, Ling FENG ${ }^{1 \#}$ \\ ${ }^{1}$ Department of Obstetrics and Gynecology, Tongji Hospital, Tongji Medical College, Huazhong University of Science and \\ Technology, Wuhan 430030, China \\ ${ }^{2}$ Department of Geriatrics, Tongji Hospital, Tongji Medical College, Huazhong University of Science and Technology, Wuhan \\ 430030, China \\ ${ }^{3}$ Department of Thoracic Surgery, Tongji Hospital, Tongji Medical College, Huazhong University of Science and Technology, \\ Wuhan 430030, China \\ ${ }^{4}$ Center Primary School in Xichuan Town, Qin'an County, Tianshui 741600, China \\ ${ }^{5}$ Department of Rehabilitation Medicine, Zhongnan Hospital of Wuhan University, Wuhan 430071, China
}

(C) Huazhong University of Science and Technology 2021

\begin{abstract}
Summary: The coronavirus disease 2019 (COVID-19) outbreak has been brought under control through a nationwide effort, and now it has become a global pandemic and the situation seems grim. We summarized the measures taken in Wuhan and analyzed the effects to comprehensively describe the factors involved in controlling the COVID-19 in China. In China, several measures such as the lockdown of Wuhan, restriction of traffic and communities, increasing hospital beds, nationwide support from medical staff, epidemic prevention equipment and supplies, and establishment of makeshift shelter hospitals have been taken. The lockdown of Wuhan reduced the propagation of cases to other cities in Hubei province and throughout China, traffic and community restrictions reduced the flow of population and the spread of disease, increasing wards and beds and medical personnel reduced the incidence of severe cases and mortality, the establishment of the Fangcang shelter hospitals provided a good isolation and monitoring environment, and further reduced the spread and fatality of the disease. The fact that China was able to control the spread of COVID-19 within three months without a specific drug or vaccine suggests that these measures are more adequate and effective.
\end{abstract}

Key words: COVID-19; mainland China; government intervention measures; healthcare

The main clinical characteristics of pneumonia are fever, cough and dyspnea, with CT showing ground-glass changes $^{[1-3]}$. In early January, 2020, the pathogenic virus was isolated and identified as coronavirus (2019-nCoV). Later, the WHO officially named the virus severe acute respiratory syndrome coronavirus 2 (SARS-CoV-2), and the resulting disease was termed as the coronavirus disease 2019 (COVID-19) ${ }^{[4]}$. During this period, the Chinese government paid close attention to the spread of the virus and infected patients. It was proven that human-to-human transmission exists ${ }^{[5]}$. The main route of transmission is respiratory tract droplet and fecaloral transmission ${ }^{[6]}$. COVID-19 is classified as class B infectious disease and managed as class A. Due to large density and big flow of population, weak precautions

Xing XIN, E-mail: M200975613@163.com; Shu-fang LI, E-mail: 409833124@qq.com

${ }^{\dagger}$ The authors contributed equally to this work.

\#Corresponding authors, Ling FENG, E-mail: fltj007@163. com; Shao-shuai WANG, E-mail: colombo2008@sina.com due to lack of awareness of people, strong infectivity and long latency of this virus, COVID-19 rapidly spread in the early days ${ }^{[7,8]}$. The Chinese authorities immediately undertook intervention measures to prevent the further spread of the epidemic and secure valuable time for China and the rest of the world. By the lockdown of Wuhan city, restricting travel in Hubei, setting up makeshift (Fangcang) shelter hospitals and providing national support to Hubei, all confirmed patients were treated in designated hospitals, and the suspected patients and COVID-19 close contacts were isolated and placed under medical observation ${ }^{[9]}$. Hence, these measures proved to be adequate with the control of the COVID-19 epidemic domestically.

At the initial stage, the number of COVID-19 patients was rising faster than the number of designated hospitals and beds available for treatment. The medical system was overwhelmed and even some local healthcare workers in Wuhan were infected. The existing measures were not enough to curb the spread of COVID-19 epidemic. People were notified of person- 
to-person transmission on Jan. 20, 2020, and Wuhan city was locked down on Jan. 23, 2020, road traffic was restricted on Jan. 26, 2020, and no community resident travels were allowed unless necessary on Feb. 10, 2020. All of the above measures were effective in blocking the route of transmission. Meanwhile, more designated hospitals and Fangcang hospitals were set up for COVID-19 patients with supporting medical staff and equipment throughout the country which reduced the rate of severe cases and death, and further reduced transmission, especially family cluster infections ${ }^{[9]}$. Here we shall further present the said measures in more detail.

\section{A MULTI-LEVEL RESTRICTION ON THE MOVEMENT OF PEOPLE TO RESTRAIN COVID-19 EPIDEMIC}

Wuhan was locked down on Jan. 23, 2020, which effectively limited the communication density and flow of people (fig. 1A ${ }^{[10]}$. Effective reproduction number (Rt) was reduced due to the lockdown of Wuhan and fell further after closed management of the community (fig. 1A ${ }^{[11]}$. Statistical results of outgoing population between Jan. 11 and Jan. 23, 2020 revealed that $67.43 \%$ of the people moved to other cities in Hubei province and $32.57 \%$ of them moved to other provinces ${ }^{[12]}$. That is one of the reasons reflecting the lesser cumulative COVID-19 patients in other provinces than other cities in Hubei including Wuhan (fig. 1B). In addition, as a result of a significant increase in the number of confirmed cases due to clinical diagnostic criteria published on Feb. 12, the number of confirmed and suspected cases gradually declined thereafter, and the number of daily confirmed cases was lower than 1000 after Feb. 19 (fig. 1C and 1D). Traffic and community restrictions and wearing of face mask by all of Wuhan people effectively reduced

$$
\begin{aligned}
& \text { - } 2019 \text { year } \\
& =2020 \text { year } \quad \text { Oversea returnees 14-day medical observation } \\
& \text { - Rt }
\end{aligned}
$$

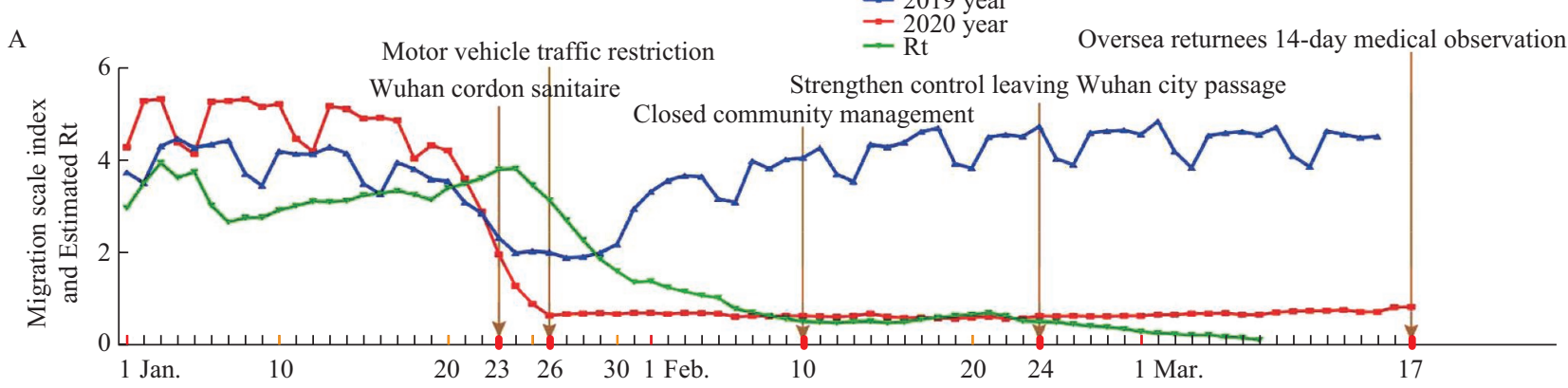

B
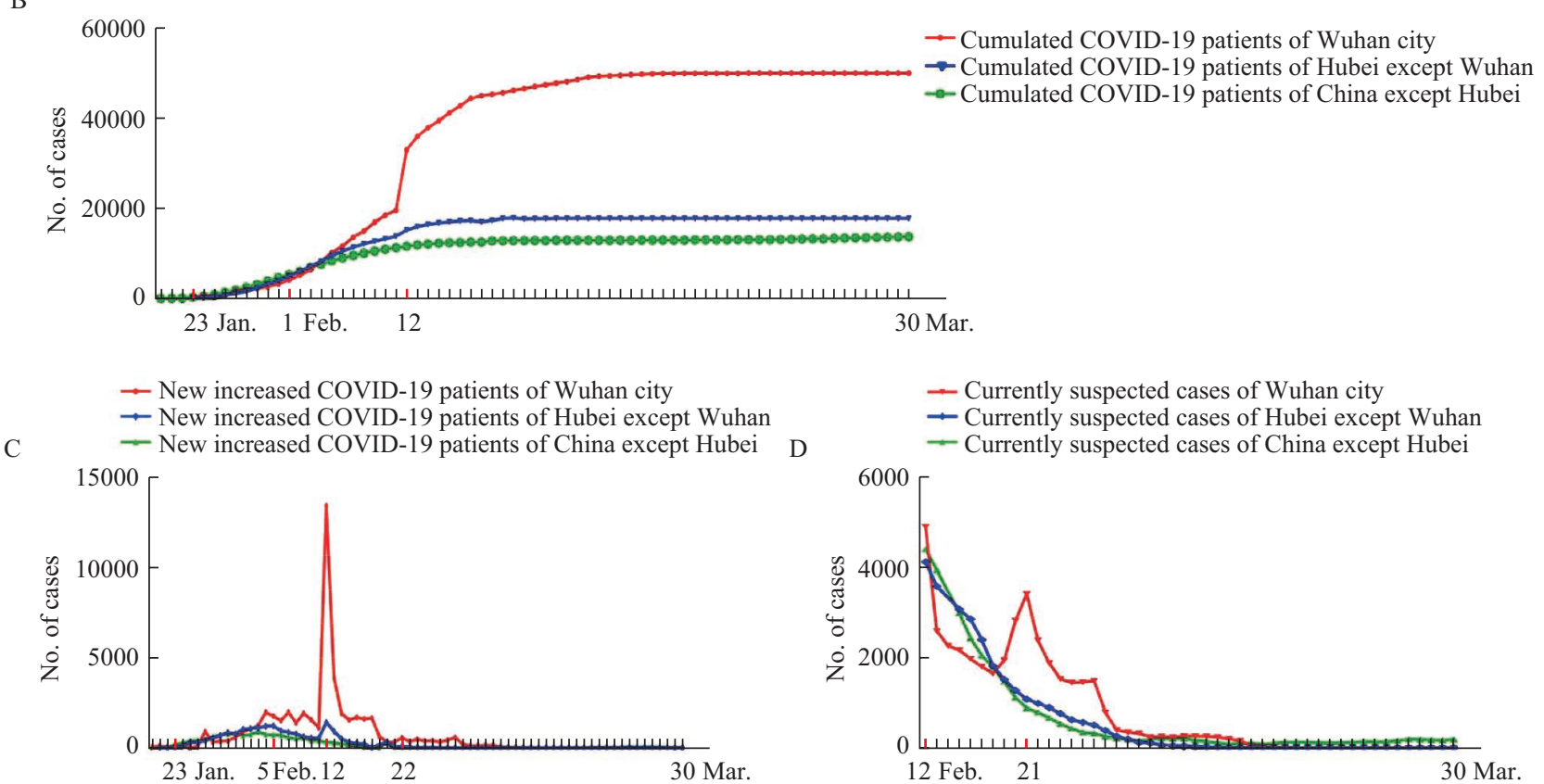

Fig. 1 Real-time human mobility data (Baidu migration scale index, http://qianxi.baidu.com/) represent fluxes of human movement of 2019 (blue line) and 2020 (red line) ${ }^{[13]}$. Chinese authorities took continuous intervention measures to restrict movement of people to control COVID-19 epidemic (dark yellow arrow). Estimates of the effective reproduction number (Rt) for laboratoryconfirmed COVID-19 patients (green line. Note: data from the article titled: Association of Public Health Interventions with the Epidemiology of the COVID-19 Outbreak in Wuhan, China. PMID: 32275295) (A) ${ }^{[11]}$. Cumulated cases of COVID-19 patients (B), new cases of COVID-19 patients (C), and currently suspected cases (D) of Wuhan city (red line), Hubei province except Wuhan city (blue line), and China except Hubei province (green line) 
the flow of population in the city and the spread of disease respectively ${ }^{[13]}$. Due to inadequate capabilities of SARS-CoV-2 nucleic acid test in the early period, the government quickly modified the diagnosis and treatment plan, which confirmed the COVID-19 cases according to the clinical diagnosis [the Guidelines for diagnosis and management of COVID-19 (5th edition, in Chinese) issued by the National Health Commission of China $]^{[14]}$. The number of clinically confirmed cases had greatly increased, enabling patients to receive treatment early to reduce severe and death cases. This measure effectively managed and isolated the clinically diagnosed COVID-19 patients.

All of these measures effectively slowed down the rate and reduced the possibility of human-tohuman transmission, and quickly cut off the source of infection. The valuable buffer time has been gained for COVID-19 follow-up treatment and the implementation of effective measures.

\section{INCREASED HOSPITAL ADMISSIONS FOR COVID-19 PATIENTS}

In order to improve medical care capacity, three measures were taken, which were comprised of building two new infectious disease hospitals, transforming general wards into infectious disease wards and providing supporting medical staffs from the whole country. Nine hospitals with about 4000 beds were designated on Jan. 25, 2020. Twenty-eight hospitals with 9027 beds were requisitioned on Jan. 28, 2020 and 48 hospitals with 22681 beds were available on Feb. 22, 2020 (fig. 2A and 2B).

Sufficient beds for COVID-19 patients were promptly provided and effective treatment which lowered the rate of severe/critical cases was given in confirmed cases (fig. 2C and 2D) ${ }^{[11]}$. On Feb. 19, 2020, the number of severe COVID-19 patients in Wuhan reached 9689, and then decreased gradually (fig. 2D). Crude death rate also decreased after these measures, the lowest death rate of $2.82 \%$ appeared on Feb. 12, 2020 with a large number of clinically diagnosed patients, and it then climbed a little higher later due to the slowed growth of cumulative confirmed patients (Crude fatality rate $=$ Cumulative deaths/Cumulative confirmed cases $\times 100 \%$ ) (fig. $2 \mathrm{E}$ ).

The cumulative number of cured patients for Wuhan city, the rest of the Hubei province, and the remaining of mainland China, reached 46002,17 151, and 12899 respectively (fig. 3A), and the cumulative number of death patients was 2548, 639, and 118, respectively on Mar. 30, 2020 (fig. 3C). The highest number of newly cured patients in Wuhan city was 2498 on Feb. 27, 2020, that in Hubei province except Wuhan
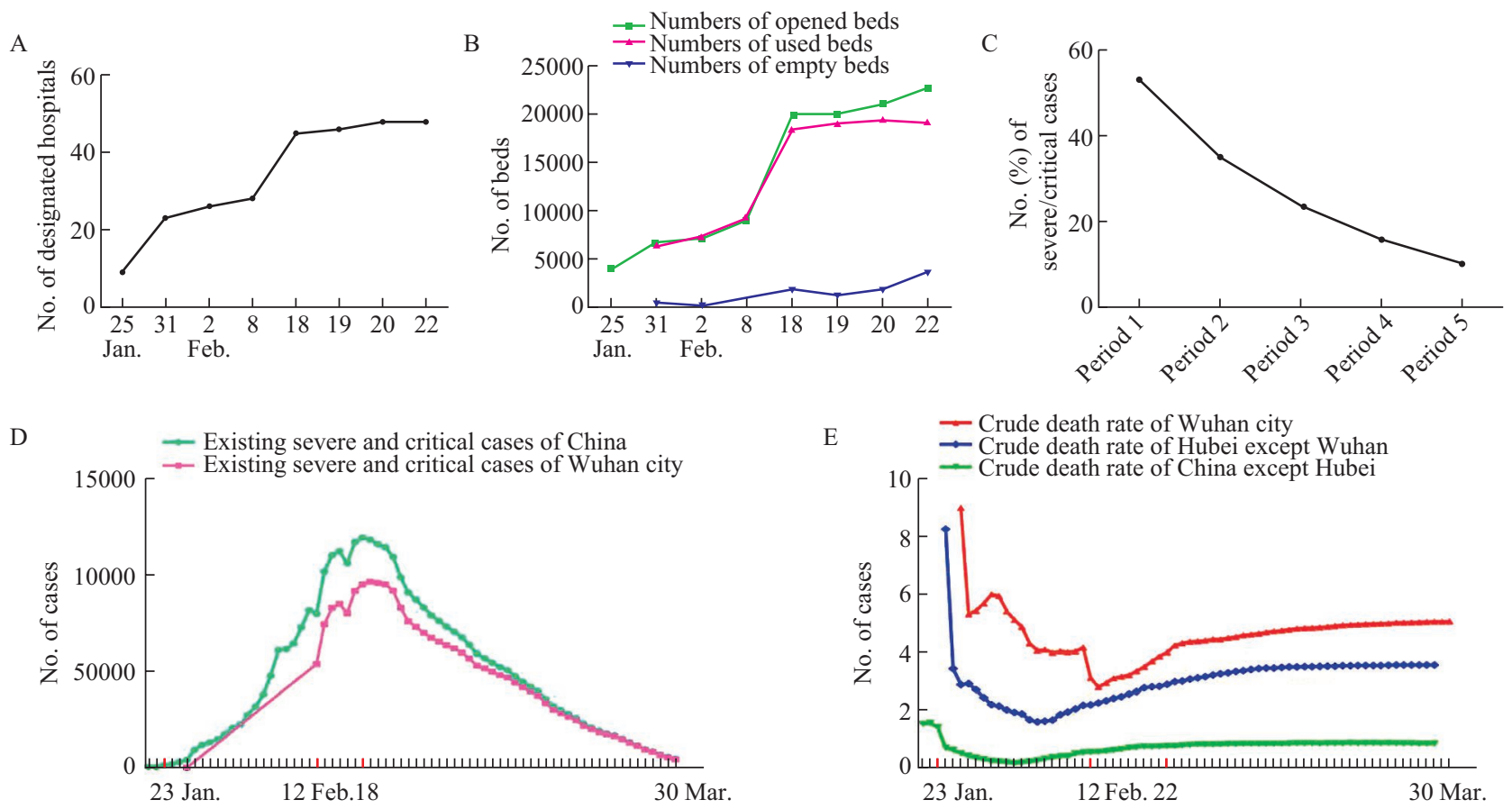

Fig. 2 Number of designated hospitals (A) and number of beds (B) from Jan. 25 to Feb. 22, 2020. Green, pink, and blue lines represent number of all available beds, used beds, and empty beds, respectively. The proportion of severe and critical cases in confirmed cases at 5 time periods (period 1, Dec. 8, 2019 to Jan. 9, 2020; period 2, Jan. 10 to 22, 2020; period 3, Jan. 23 to Feb. 1, 2020 ; period 4, Feb. 2 to 16, 2020; period 5, Feb. 17 to Mar. 8, 2020. Note: data from the article titled: Association of Public Health Interventions with the Epidemiology of the COVID-19 Outbreak in Wuhan, China. PMID: 32275295) (C) ${ }^{[1]}$. Existing severe and critical cases of China (light green line) and Wuhan city (pink line) from Jan. 21 to Mar. 30, 2020 (D). Crude death rate of Wuhan city (red line), Hubei province except Wuhan city (blue line), and China except Hubei province (green line) from Jan. 21 to Mar. 30, 2020 (E) 

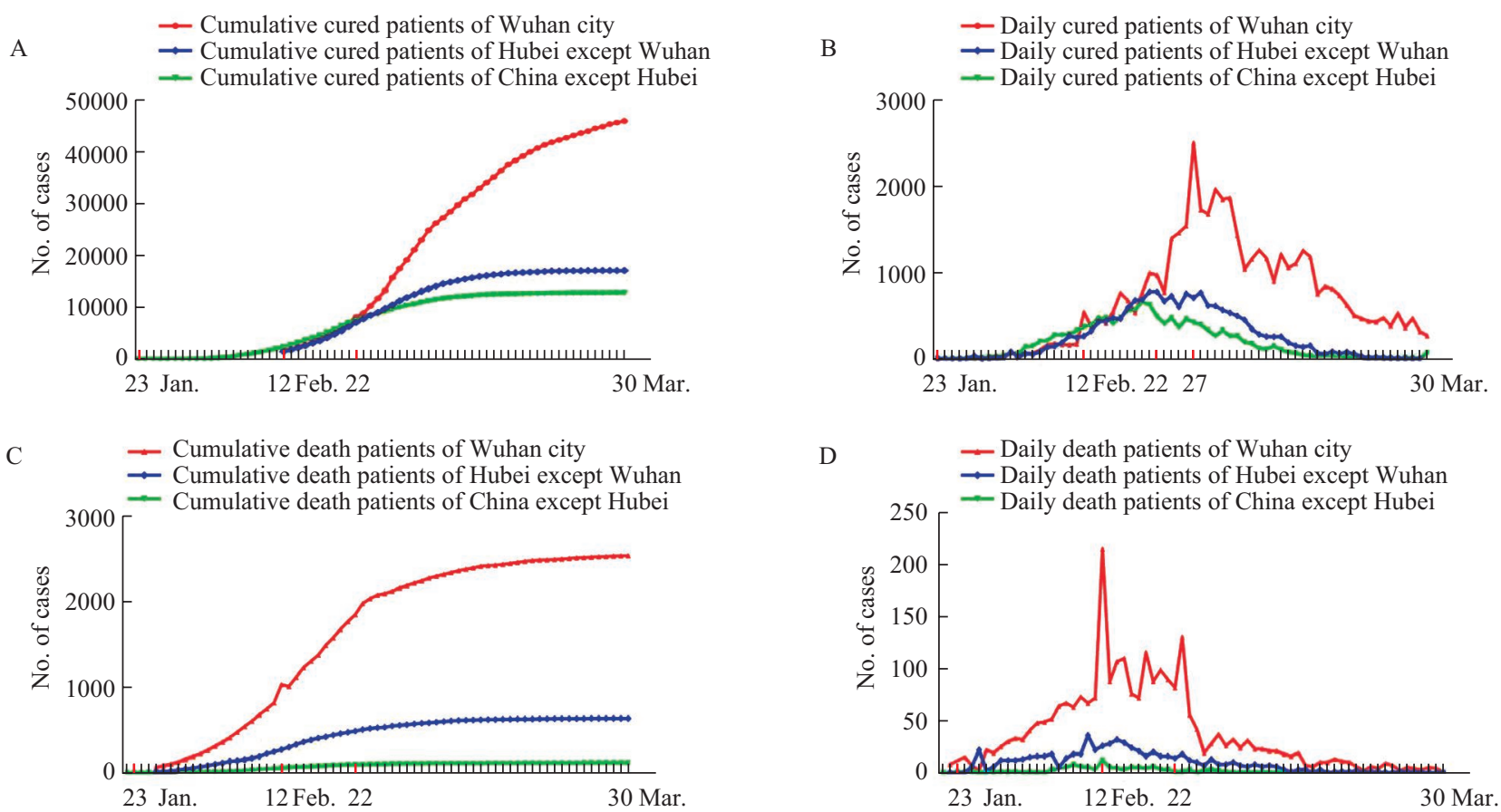

Fig. 3 Cumulative cured patients (A), daily cured patients (B), cumulative death rate (C), and daily death reports (D) of Wuhan city (red line), Hubei province except Wuhan city (blue line), and China except Hubei province (green line)

city reached 777 cases on Feb. 22, 2020, and that in mainland China except Hubei province reached 658 cases on Feb. 20, 2020 (fig. 3B). The highest number of new deaths in Wuhan city and mainland China except Hubei province was 216 and 12 respectively on Feb. 12, 2020, and that in Hubei province except Wuhan city was 12 cases on Feb. 10, 2020 (fig. 3D). Until Mar. 30,2020 , the cumulative number of cured patients in Wuhan continued to accelerate, while the cumulative number of deaths increased relatively smoothly. The increases of designated hospitals and beds in Wuhan city achieved effective treatment of COVID-19 patients and reduced the rate of severe/critical cases and death cases in confirmed patients. Moreover, with the increase of designated hospitals, COVID-19 patients had beds to receive treatment, which could fully reduce the panic of patients, and conduct unified management of COVID-19 patients to prevent cross-infection and larger spread of infection.

\section{STRENGTHENING THE ISOLATION OF SUSPECTED CASES AND CONTACTS AND MILD PATIENTS}

Due to the large population density and inadequate isolation conditions in Chinese homes, there were more and more confirmed and suspected patients. Besides, civilians isolated at home could not carry out optimal self-monitoring and accurately identify the situation of severe/critical patients. Hence, the government carried out a great approach which was the establishment of Fangcang shelter hospitals. These makeshift hospitals provided plenty of beds for mild and moderate patients with COVID-19, which isolated them from their family members and communities, while providing medical care, disease surveillance and essential living and social engagement ${ }^{[9]}$.

On Feb. 5, 2020, the first Fangcang shelter hospital was built to treat patients, and a total of more than 30 temporary hospitals were established in Wuhan city, and 16 were still in operation until Feb. 22, 2020. By Feb. 22, 2020, there were 13148 available beds in the Fangcang shelter hospitals, of which 9274 had been used and 3874 were empty (fig. 4A). The last Fangcang shelter hospital was closed on Mar. 10, 2020.

Contacts and suspected cases were under medical observation at designated isolation observation place, such as designated hotels. A total of 706017 close contacts were followed up until Mar. 30, 2020, and the number of people undergoing medical observation reached its peak on Feb. 7, 2020, with a total of 189660 cases. 31124 cases of close contacts disengaged from medical observation which reached a peak on Feb. 8, 2020 (fig. 4B). The number of newly suspected cases have gradually declined since Feb. 12, 2020 and have not exceeded 1000 per day in Wuhan city. No new suspected cases were signaled in Wuhan city after Mar. 16, 2020 (fig. 4C).

With the full operation of Fangcang shelter hospitals, the growth rate of cumulative COVID-19 patients in Wuhan city continued to decline after Feb. 12, 2020 (fig. 4D). The cumulative growth rate of COVID-19 patients in China excluding Hubei province continued to decline after Jan. 23, 2020 (fig. 4E). 


\section{GUARANTEE FOR THE LIVELIHOOD OF THE PEOPLE OF WUHAN}

In China, COVID-19 patients were not required to pay for their therapy, which greatly alleviated their concerns about medical expenses. Therefore, more COVID-19 patients were willing to be hospitalized to receive more specialized treatment, which reduced the exposure of COVID-19 patients to their family members and general population. At the same time, in spite of restricting traffic and closing communities, safeguards from Chinese authority were provided, such as vehicles for emergency medical transfer and food distribution by community group. Everybody involved in this aspect of the epidemic tried their best to protect people's life and reduce the transmission of SARSCoV-2.

\section{COVID-19 EPIDEMIC PREVENTION AND CONTROL OF PERSISTING CHALLENGES}

\subsection{Management of Overseas Returnees}

COVID-19 epidemic control has achieved staged victory in China, but difficulties still surfaced at every phase. With the spread of COVID-19 epidemic worldwide, some overseas nationals are returning to China, that may cause another large-scale outbreak in every province of China. To prevent the COVID-19 reepidemic and outbreak, the Chinese government has decided to conduct the strategy of imposing a 14-day health isolation and observation for oversea returnees, with the aim of screening out COVID-19 patients. On Mar. 4, 2020, there were 139 new COVID-19 patients, of which 2 cases were oversea returnees (fig. 5A). Since Mar. 30, 2020, there have been up to 771 oversea returnees diagnosed with COVID-19 (fig. 5B).

\subsection{Untested Asymptomatic COVID-19 Patients}

Asymptomatic patients infected with SARSCoV-2 have been sources of infection and are liabilities after the re-opening of Wuhan city. The team of Professor WU Tang-chun from Tongji Medical College used the most conservative model to predict that $59 \%$ of asymptomatic COVID-19 patients may go undetected in China ${ }^{[15]}$. Statistical analysis of the Chinese Center for Disease Control and Prevention revealed that a total of 72314 cases were reported in mainland China, with 44672 confirmed COVID-19 patients (61.8\%), 16186 suspected cases (22.4\%), 10567 clinically diagnosed cases $(14.6 \%)$ and 889 asymptomatic infections (1.2\%). The proportion of asymptomatic infections excluding suspected cases was about $1.6 \%$. There were 130 new asymptomatic COVID-19 patients and a total of 1367 asymptomatic COVID-19 patients on Mar. 31, 2020. Now, an epidemiological study is underway to evaluate the exact scale of asymptomatic COVID-19 patients.

\section{DISCUSSION}

The COVID-19 epidemic spread on a large scale because of the limited understanding of the
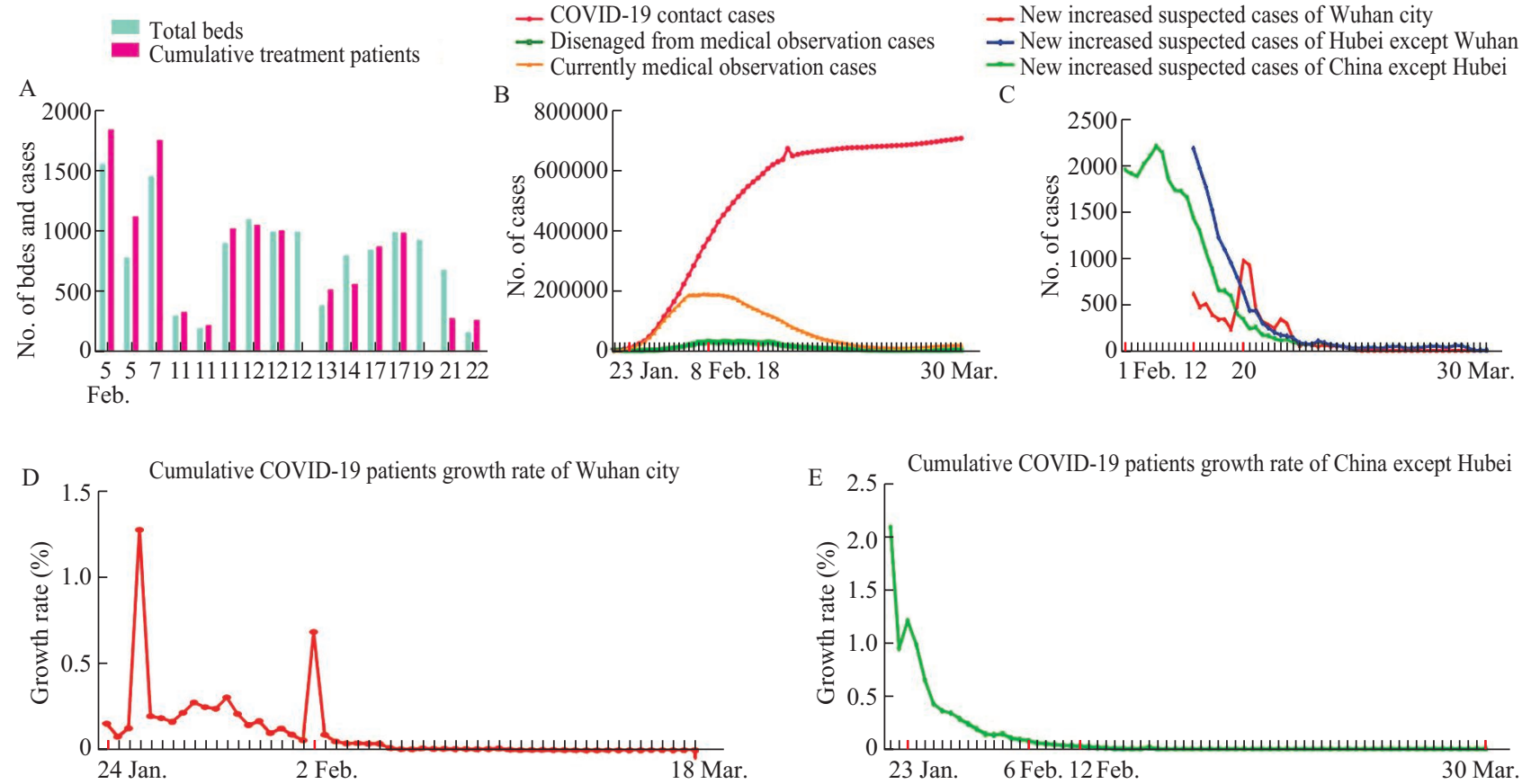

Fig. 416 Fangcang shelter hospitals opened from Feb. 5 to Feb. 22, 2020. Every Fangcang shelter hospital showed total beds available (light green) and cumulative patients being treated (pink) (A). COVID-19 contact cases (red line), disengaged from medical observation cases (green line), and currently under medical observation cases (orange line) showed from Jan. 20 to Mar. 30, 2020 (B). New suspected cases in Wuhan city (red line), Hubei province except Wuhan city (blue line), and China except Hubei province (green line) (C). Growth rate of cumulative COVID-19 patients in Wuhan city (D) and China except Hubei province (E) 

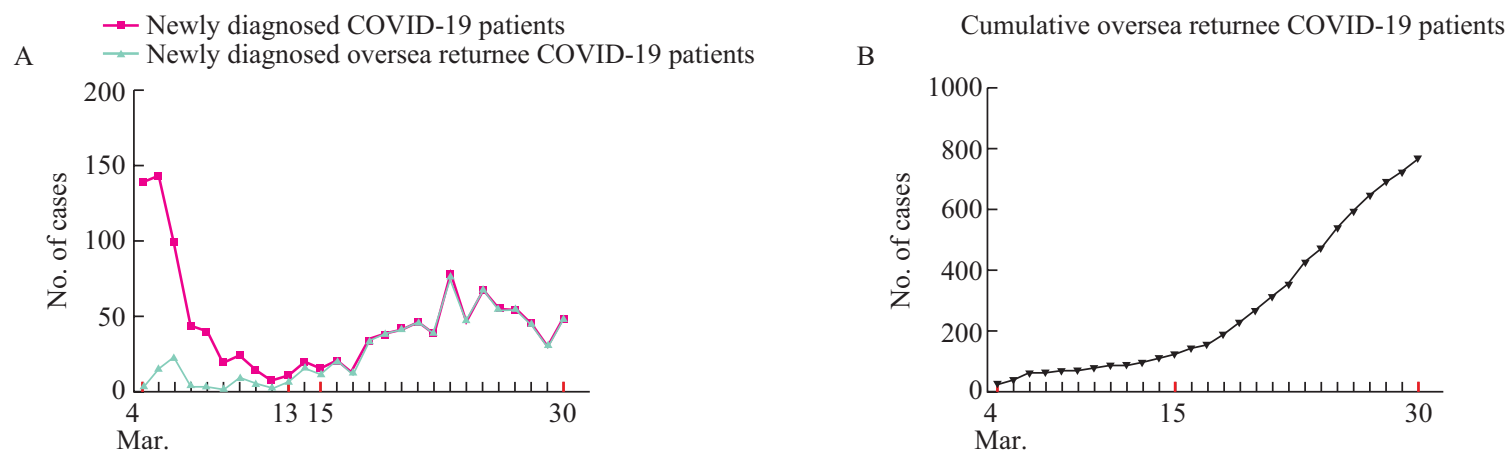

Fig. 5 All of newly diagnosed COVID-19 patients (red line) and newly diagnosed oversea returnee COVID-19 patients (light green) between Mar. 4 to Mar. 30, 2020 (A). Cumulative oversea returnee COVID-19 patients from Mar. 4 to Mar. 30, 2020 (B)

epidemiological characteristics of COVID-19 at the beginning. The Chinese government immediately issued relevant regulations and strategies, and the COVID-19 epidemic was effectively controlled in late Feb. 2020. By predictive models, Liu et al believed that if no interventions were implemented, the peak period of the epidemic would be at the end of April. Millions of people would be infected by SARS-CoV-2 and the maximum number of the existing cases could be more than 850000 in Wuhan city ${ }^{[12]}$. Most notably, restriction on mobility (called cordon sanitaire) was imposed on Wuhan city on Jan. 23, 2020. The Baidu migration index almost reached its lowest when motor vehicles and traffic were restricted on Jan. 26, 2020 ${ }^{[13]}$. Based on the COVID-19 incubation period of 14 days, daily increase of infected patients reached its peak on Feb. 11, 2020, while our statistics showed this peak happened on Feb. 12, 2020. Of course, the sudden increase in the number of newly diagnosed patients on Feb. 12, 2020 is also related to improvements in COVID-19 detection ability. The peak of newly diagnosed patients actually appeared earlier than the prediction model, which supports the effectiveness of quarantine and control measures ${ }^{[12,16]}$.

With the surge of COVID-19 patients, doctors and nurses in Wuhan city were struggling while being exposed to COVID-19 for a long time and inadequate prevention equipment, and more than 3000 healthcare workers were infected in the early stage. With the dispatch of healthcare workers from other provinces of China to support Wuhan city, exposure time in COVID-19 atmosphere was shortened, local healthcare workers infections became rare and none of the healthcare workers from other provinces were infected ${ }^{[17]}$. By rapidly increasing the number of designated hospitals, more COVID-19 patients were effectively isolated, treated and the COVID-19 epidemic was brought under control. Psychological help is important to comfort the mind, reduce panic and plays a crucial role in social stability.

To relieve pressure of designated hospitals and effectively isolate COVID-19 patients, makeshift shelter hospitals were implemented for the first time in China to tackle COVID-19. Fangcang shelter hospitals isolated COVID-19 patients more effectively than home isolation. It has been reported that $75 \%-80 \%$ of the cluster transmission occurred in families in China, suggesting high rate of intrafamilial transmission ${ }^{[9,18,19]}$. The Fangcang shelter hospital consisted of professional doctors and nurses who provided basic medical care and real-time monitoring of the disease progression. If the COVID-19 patients' general condition worsened, they would be immediately transferred to a designated hospital and, under these conditions, the time from the onset of severe symptoms to admission to a higherlevel designated hospital was substantially reduced.

Owing to the effective interventions carried out by the Chinese authorities, tremendous joint efforts from health care workers and the cooperation of all the Chinese people, the reproduction number (Rt) for COVID-19 cases decreased from 3.80 on Jan. 23, 2020 to 0.10 on Mar. $8,2020^{[11]}$. However, there are currently three challenges in controlling COVID-19 epidemic in China: firstly, management of COVID-19, recovery and discharge of patients; secondly, management of oversea returnees; thirdly, the screening of asymptomatic and mild cases of COVID-19. COVID-19 nucleic acid (NA) test may be positive again in cured patients who do not have any symptoms. Cured and discharged patients still need a specified place for isolation, close monitoring and follow-up. The low positive rate of COVID-19 NA tests and long incubation period of COVID-19 may be caused by oversea returnees and asymptomatic patients scattered around other provinces of China, which can potentially lead to another large-scale COVID-19 outbreak in China. Increasing evidence has suggested that asymptomatic and pre-symptomatic cases can be infectious to the susceptible population ${ }^{[18,20]}$. Strengthening the management of oversea returnees, detecting asymptomatic patients as soon as possible are very important determinants for COVID-19 epidemic control in ending stage. At present, there is no obvious 
increase in the number of new COVID-19 patients. It is to be noted that Wuhan city mobility has gradually returned to normal, and the communities have been opened. As Wuhan city gradually returns to normal as usual, we still face some challenges and need to be vigilant and ensuring optimal defenses. COVID-19 is the common enemy of all mankind and we need to help each other in this disaster. We thereby share our data and experience to assist more people around the world.

\section{Acknowledgments}

We are very grateful to the National Health Commission of the People's Republic of China, Health Commission of Hubei Province and Wuhan Municipal Health Commission for providing reliable source of information.

\section{Conflict of Interest Statement} interest.

The authors declare that they have no conflict of

\section{REFERENCES}

1 Hui DS, Azhar EI, Madani TA, et al. The continuing 2019-nCoV epidemic threat of novel coronaviruses to global health - The latest 2019 novel coronavirus outbreak in Wuhan, China. Int J Infect Dis, 2020,91:264266

2 Wang D, Hu B, Hu C, et al. Clinical Characteristics of 138 Hospitalized Patients with 2019 Novel Coronavirus-Infected Pneumonia in Wuhan, China. JAMA, 2020,323(11):1061-1069

3 Shi H, Han X, Jiang N, et al. Radiological findings from 81 patients with COVID-19 pneumonia in Wuhan, China: a descriptive study. Lancet Infect Dis, 2020,20(4):425-434

4 Rockx B, Kuiken T, Herfst S, et al. Comparative pathogenesis of COVID-19, MERS, and SARS in a nonhuman primate model. Science, 2020,368(6494): 1012-1015

5 Chan JF, Yuan S, Kok KH, et al. A familial cluster of pneumonia associated with the 2019 novel coronavirus indicating person-to-person transmission: a study of a family cluster. Lancet, 2020,395(10223):514-523

6 Xiao F, Tang M, Zheng X, et al. Evidence for Gastrointestinal Infection of SARS-CoV-2. Gastroenterology, 2020,158(6):1831-1833.e3

7 Zhao S, Lin Q, Ran J, et al. Preliminary estimation of the basic reproduction number of novel coronavirus
(2019-nCoV) in China, from 2019 to 2020: A datadriven analysis in the early phase of the outbreak. Int $\mathrm{J}$ Infect Dis, 2020,92:214-217

8 Zhu N, Zhang D, Wang W, et al. A Novel Coronavirus from Patients with Pneumonia in China, 2019. N Engl J Med, 2020,382(8):727-733

9 Chen S, Zhang Z, Yang J, et al. Fangcang shelter hospitals: a novel concept for responding to public health emergencies. Lancet, 2020,395(10232):1305-1314

10 Kraemer MUG, Yang CH, Gutierrez B, et al. The effect of human mobility and control measures on the COVID-19 epidemic in China. Science, 2020,368(6490):493-497

11 Pan A, Liu L, Wang C, et al. Association of Public Health Interventions with the Epidemiology of the COVID-19 Outbreak in Wuhan, China. JAMA, 2020,323(19):19151923

12 Liu M, Ning J, Du YR, et al. Modeling the Evolution Trajectory of COVID-19 in Wuhan, China: Experience and Suggestions. Public Health, 2020,183:76-80

13 Baidu migration index. http://qianxi.baidu.com/, Accessed on March 11, 2020.

14 New coronavirus pneumonia prevention and control program (5th ed.) (Chinese). 2020. Available at: http:// www.gov.cn/zhengce/zhengceku/2020-02/05/5474791/ files/de44557832ad4be1929091dcbcfca891.pdf.

15 Wang CL, Liu L, Hao XJ, et al. Evolving Epidemiology and Impact of Non-pharmaceutical Interventions on the Outbreak of Coronavirus Disease 2019 in Wuhan, China. Available at: https://www.medrxiv.org/content/1 0.1101/2020.03.03.20030593v1.

16 Yang Z, Zeng Z, Wang K, et al. Modified SEIR and AI prediction of the epidemics trend of COVID-19 in China under public health interventions. J Thorac Dis, 2020,12(3):165-174

17 Adams JG, Walls RM. Supporting the Health Care Workforce During the COVID-19 Global Epidemic. JAMA, 2020,323(15):1439-1440

18 Tong ZD, Tang A, Li KF, et al. Potential Presymptomatic Transmission of SARS-CoV-2, Zhejiang Province, China, 2020. Emerg Infect Dis, 2020,26(5):1052-1054

19 Li P, Fu JB, Li KF, et al. Transmission of COVID-19 in the terminal stage of incubation period: a familial cluster. Int J Infect Dis, 2020,96:452-453

20 Li C, Ji F, Wang L, et al. Asymptomatic and Humanto-Human Transmission of SARS-CoV-2 in a 2-Family Cluster, Xuzhou, China. Emerg Infect Dis, 2020,26(7): 1626-1628

(Received May 14, 2020; accepted Aug. 3, 2020) 